diffuse away from that position, it will remain a hot spot.

There are many precedents for such theorems in the geometry of the Laplace operator, whence the conjecture. In forthcoming work, David Jerison and Nikolai Nadirashvili prove that the hot spots conjecture is true for any convex domain. R. Bañuelos and Burdzy have proved it for certain long, thin domains, not necessarily convex ones (J. Funct. Anal., in the press).

Nevertheless, Burdzy and Werner show that the full conjecture is false (they also say that Jerison and Nadirashvili have a different proof of this). Their basic idea is to consider a domain that resembles a bicycle wheel: a hub, at least three long thin spokes, and a tyre. Start with the hub hot and the tyre cold. Think about the flow of cold rather than that of heat. Cold reaches the hot hub only through the thin spokes so, as time passes, the hottest spot is pushed towards the centre of the hub.

So far, so good: the trick is to prove all this. Burdzy and Werner first define a domain with three identical spokes each about 20 times as long as they are wide, a hub and a tyre. Unlike a real bicycle wheel, however, the spokes extend well outside the tyre - and the actual shape has to be chosen very carefully for the ideas of the proof to work. This domain is a complicated polygon with the symmetry of an equilateral triangle: three rotations and three reflections. The domain has three holes, each bounded by part of the hub, part of the tyre and parts of two spokes. The symmetry makes it possible to use known results about eigenfunctions in symmetric domains at crucial stages of the proof. However, the actual domain to which Burdzy and Werner's results apply differs slightly: a cut is made through the domain just described, to reduce the number of holes to two.

Whether the hot spots conjecture is true for domains with only one hole, or none, remains open. The geometry of the Laplace operator does not reveal its secrets lightly, but it undoubtedly remains a hot topic.

Ian Stewart is in the Department of Mathematics,

University of Warwick, Coventry CV4 7AL, UK.

e-mail:ins@maths.warwick.ac.uk

Ecology

\title{
Scaling, energetics and diversity
}

\author{
Robert J. Whittaker
}

$\mathrm{T}$ here are few established laws in ecology. Most ecologists have to contend with systems of bewildering complexity, in which it is hard to separate the wood from the trees. Scientific disciplines without a good framework of rules and laws are generally considered the poor relations of their ilk, and, as human impacts on our planet appear to have reached alarming levels, the need for reliable ecological theory is hard to overstate. The discovery and demonstration of new laws in ecology - such as the Universal Growth Law for plants described by Enquist et al. ${ }^{1}$ on page 907 of this issue - is thus particularly noteworthy.

The existence of power relations (allometric relationships), whereby biological variables tend to increase or decrease in relation to body mass raised to the power of a quarter or three quarters, has been known for over half a century. But only recently has the realization of the ubiquity and generality of this relationship, which occurs at many levels of biological organization, elevated it to the status of a biological law2. One of the best-known quarter-power scaling relationships is that between body mass and metabolic rate in animals, which it now appears holds over an extraordinary 27 orders of magnitude of size ${ }^{2}$. Enquist and

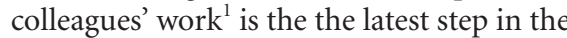
determination of analogous allometric relationships for plants, with potentially wide- ranging implications for ecological science.

Enquist and colleagues' general case is that production within species declines in a uniform fashion with increasing size. Why this should be so, theoretically, can be explained in relation to the energy and materials essential to all biological processes. In previous analyses of allometric relationships in plants, reported in Nature earlier this year ${ }^{3}$, this team has shown how plants conform to a suite of scaling rules, and constitute distribution networks with a fractal-like (that is, self-similar) architecture. Physical and biological constraints on the distribution of resources (water and nutrients in solution) through these networks underlie the ' $3 / 4$ power scaling' of metabolic rate with mass $\left(\right.$ metabolic rate $=$ mass $\left.^{0.75}\right)$. Put another way, biological rates are ultimately limited by the rates at which energy and materials can be distributed between the surfaces where they are exchanged and the tissues where they are used.

Enquist et al. provide a demonstration of their theoretical model drawn from a sample of 2,283 trees of 45 species, measured at two points in time, 20 years apart, from seasonally dry tropical forest in Costa Rica. This shows that a large array of different species show the expected relationship of diminishing growth with increasing size. The biology and ecology of trees is, of course, highly variable - there are light-demanding and

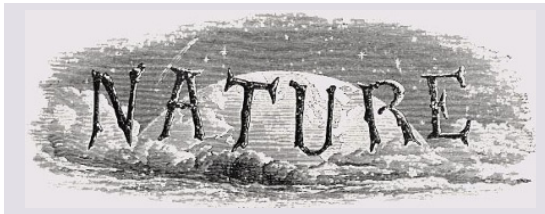

100 YEARS AGO

The long-standing question as to the admittance of women into full fellowship of scientific societies was brought before a meeting of the Lady Warwick Agricultural Association for Women on Thursday last, and the following resolution, supported by a paper by Mrs. Farquharson, was adopted:

"That it is desirable and important that duly qualified women should have the advantages of full fellowship in scientific and other learned societies, e.g. the Royal, the Linnean and the Royal Microscopical." The arguments in favour of and in opposition to this proposal have been stated so many times that most members of scientific societies are familiar with them. Six years ago the council of the Royal Geographical Society elected several ladies as fellows, but their action was disapproved at two special meetings, and resolutions to the effect that it was inexpedient to admit ladies as ordinary fellows were carried by conclusive votes. ... The time may of course come when women will be equally eligible with men for membership of the learned societies, but facts such as those cited show that there is distinct opposition to the admittance of women at present, and no sudden change of feeling can be expected, though individual cases of "duly qualified" women might be considered.

From Nature 26 October 1899.

\section{YEARS AGO}

All through the nineteenth century and well into the twentieth, mathematicians studied the problems suggested by the generation and propagation of waves on the surface of the sea and other bodies of water. But during all this time there was very little scientific observation of waves, and practically none of it was by means of specially designed measuring instruments. Oceanographers paid very little attention to the details of the phenomena. But during the Second World War, it was part of the preparation for the landing of troops and material on beaches that forecasts should be made of the state of swell and surf on those beaches at particular times... C.C. Bates, of the United States Navy, gives an account of the utilization of wave forecasting in the invasions of Normandy, Burma and Japan. Excluding cases in which forecasts of winds were in error, it was found that forecasts of waves on Normandy landing beaches were correct for 88 per cent of the time.

From Nature 29 October 1949. 


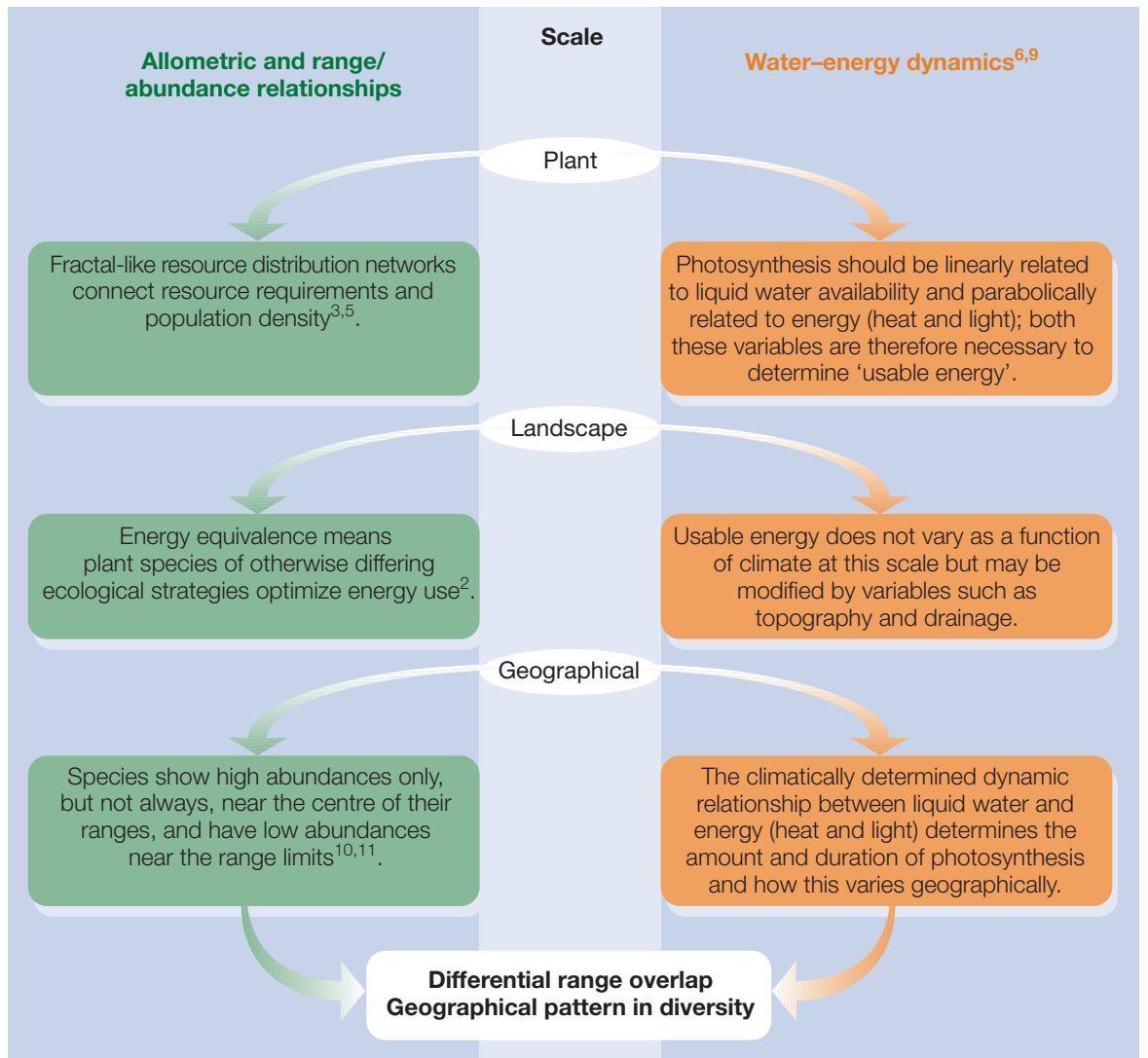

Figure 1 Macroecological insights into plant diversity. Differential range overlap produces geographical pattern (for example, latitudinal gradients) in diversity, wherein the species capable of persisting through time in a region are those able to optimize energy use ${ }^{1}$. Climate determines the capacity for richness, consistent with richness at the macroscale being monotonically related to increasing usable energy (that is, productivity) and parabolically related to climatic energy ${ }^{6}$.

shade-tolerant species, insect- and animalpollinated species, species adapted to differing soil conditions and so forth. In tropical forests, these and other traits occur in many different combinations, such that reduction of variation onto a single successional axis pioneer to 'climax' - has proven impracti$\mathrm{cal}^{4}$. Similarly, no satisfactory general theory to explain variation in diversity has emerged from the study of these traits and related processes.

Enquist et al. ${ }^{1}$ have shown, however, that when variation in wood density (itself an important trait reflecting differences in the allocation of metabolic energy) is taken into account, the data fit the theoretical power relation demanded by their model remarkably well. What this means is that usable energy is being deployed in a variety of growth-form, life-history and wood-density strategies that each amount to varying ways of attaining the same optimal use of energy. In other words, there is energy equivalence across species (see also ref. 5).

Within this seeming evolutionary constraint, there are interspecific differences in energy allocation over the lifespan. These take the form of differing rates of volume increment, differing lifespan and varying reproductive strategies, which together underpin the diversity of these tropical forests. The key

\section{Mutation}

$\mathrm{T}$ he future is unpredictable. Survival therefore depends on the generation of a large repertoire of biological diversitythe evolutionary equivalent of buying a large number of lottery tickets. Natural selection can be compared with drawing the lottery, in that a strong selection event reduces diversity because it allows only a small fraction of the original population to survive (the winners).

\section{Miroslav Radman}

findings may be useful in developing a theory of diversity which, like the diversity problem itself, is trans-scalar in nature (Fig. 1).

In practice, although Enquist and colleagues' scaling relationships are detectable within trees and in smaller plant units, even down to the cell ${ }^{2}$, the significance of energetics in regulating diversity at the local scale (for example, 0.1 hectare) is often undetectable, being obscured by other variables whose measurable heterogeneity occurs at local to landscape scales. The significance of variation in usable energy becomes evident, however, at the macro-scale, at which O'Brien's Interim General Model ${ }^{6}$ describes the CPR as a linear function of liquid water and a parabolic function of energy. Liquid water is also fundamental, not just as a vital ingredient in the process of photosynthesis, but also to the development of allometric models, as energy and nutrients are moved into and through plants in solution ${ }^{3}$.

So these scaling laws seem to provide a basis for the development of ecological theories that are trans-scalar in geographical space, something with which traditional branches of ecology have struggled. In separating the wood from the trees - at least in terms of correcting for density variations Enquist et al. have shown the vitality of the emerging field of macroecology $y^{2,7,8}$.

Robert J. Whittaker is in the School of Geography, University of Oxford, Mansfield Road, Oxford OX1 3TB, UK.

e-mail:Robert.Whittaker@geog.ox.ac.uk

1. Enquist, B. J., West, G. B., Charnov, E. L. \& Brown, J. H. Nature 401, 907-911 (1999).

2. Brown, J. H. \& West, G. B. (eds) Scaling in Biology (Oxford Univ. Press, 1999).

point in relation to diversity is that, in the block of forest studied, there is clear evidence that energetics are crucial to the persistence of the diversity of species present, otherwise their growth in biomass would not exhibit such a consistent scaling relationship. Hence systems with more usable energy should allow for a greater subdivision of energy into more distinct plant niches. This ties in with another fundamental relationship, recently reported by E. M. O'Brien in the Journal of Biogeography ${ }^{6}$ - a capacity rule describing the climatic potential for richness (CPR). Both sets of
3. West, G. B. et al. Nature 400, 664-667 (1999).

4. Brown, N. D. \& Jennings, S. in Dynamics of Tropical

Communities (eds Newbery, D. M., Prins, H. T. \& Brown, N.

D.) 79-94 (Blackwell Science, Oxford, 1998).

5. Damuth, J. D. Nature 395, 115-116 (1998).

6. O’Brien, E. M. J. Biogeogr. 25, 379-398 (1998).

7. Brown, J. H. Macroecology (Univ. Chicago Press, 1995).

8. Maurer, B. A. Untangling Ecological Complexity: The

Macroscopic Perspective. (Univ. Chicago Press, 1999).

9. O'Brien, E. M. J. Biogeogr. 20, 181-198 (1993).

10. Stevens, G. C. \& Enquist, B. J. in Ecology and Biogeography of Pinus

(ed. D. M. Richardson) 183-190 (Cambridge Univ. Press, 1998). 11.Enquist, B. J. et al. Evol. Ecol. 9, 586-604 (1995).

\section{Enzymes of evolutionary change}

Diversity then needs to be restored to allow future selections to be faced. In other words, genetic diversification needs to be a continuous process, and reports in Proceedings of the National Academy of Sciences, Molecular Cell $^{2}$ and the Journal of Biological Chemistry ${ }^{3}$ show how this might be done.

Genetic diversity is generated by mutagenesis, which causes changes in the DNA 